TRANSACTIONS OF THE

AMERICAN MATHEMATICAL SOCIETY

Volume 361, Number 11, November 2009, Pages 5721-5736

S 0002-9947(09)04566-8

Article electronically published on June 16, 2009

\title{
FUNDAMENTAL SOLUTIONS AND TWO PROPERTIES OF ELLIPTIC MAXIMAL AND MINIMAL OPERATORS
}

\author{
PATRICIO L. FELMER AND ALEXANDER QUAAS
}

\begin{abstract}
For a large class of nonlinear second order elliptic differential operators, we define a concept of dimension, upon which we construct a fundamental solution. This allows us to prove two properties associated to these operators, which are generalizations of properties for the Laplacian and Pucci's operators. If $\mathcal{M}$ denotes such an operator, the first property deals with the possibility of removing singularities of solutions to the equation
\end{abstract}

$$
\mathcal{M}\left(D^{2} u\right)-u^{p}=0, \quad \text { in } \quad B \backslash\{0\},
$$

where $B$ is a ball in $\mathbb{R}^{N}$. The second property has to do with existence or nonexistence of solutions in $R^{N}$ to the inequality

$$
\mathcal{M}\left(D^{2} u\right)+u^{p} \leq 0, \quad \text { in } \mathbb{R}^{N} .
$$

In both cases a common critical exponent defined upon the dimension number is obtained, which plays the role of $N /(N-2)$ for the Laplacian.

\section{INTRODUCTION}

In the study of the semi-linear elliptic partial differential equation

$$
\Delta u+f(u)=0 \quad \text { in } \quad \Omega \subset \mathbb{R}^{N},
$$

enormous effort has been directed towards the understanding of the role of the nonlinearity of $f$ in its interaction with the Laplacian. The case of a simple power $f(s)= \pm|s|^{p-1} s$ is very important in itself and as a model for more general $f$; thus much attention has been directed to the critical values of the exponent $p$, where changes in the structure of (1.1) take place. For $p>1$ and $N \geq 3$, the value

$$
p_{*}=\frac{N}{N-2}
$$

is critical regarding two different properties of solutions to (1.1). First, concerning solutions in a punctured ball, $B_{R} \backslash\{0\}$, with singularities at the origin and its possible removability, we have the following theorem proved by Brezis and Veron in [4].

Theorem 1.1. Assume $p>1$ and $N \geq 3$. Then the singularities of a solution to the equation

$$
\Delta u-|u|^{p-1} u=0, \quad \text { in } \quad B(0, R) \backslash\{0\}, R>0,
$$

Received by the editors January 4, 2006 and, in revised form, May 10, 2007.

2000 Mathematics Subject Classification. Primary 35J60; Secondary 35B05, 35B60.

Key words and phrases. Extremal operators, viscosity solutions, fundamental solutions, removability of singularities, Liouville-type theorems. 
are removable if and only if

$$
p \geq \frac{N}{N-2} .
$$

Removability of singularities of solutions to partial differential equations of elliptic type has attracted the attention of many authors. We mention in particular the classical work of Serrin [22, 23], Bers [2], and De Giorgi and Stampacchia [10]. We refer the reader to the monograph by Véron [25] and the paper by Labutin [18] for further references.

Second, following with the exponent $p_{*}$ given in (1.2), we have a result concerning the existence of super-solutions in $\mathbb{R}^{N}$, which have been proved by Gidas in [13,

Theorem 1.2. Assume $p>1$ and $N \geq 3$. Then there are no nontrivial supersolutions for

$$
\Delta u+u^{p-1}=0, \quad u \geq 0, \quad \text { in } \quad \mathbb{R}^{N}
$$

if and only if

$$
p \leq \frac{N}{N-2} .
$$

Keeping the power nonlinearity in equations (1.3) and (1.5) we may consider a more general operator instead of the Laplacian. Let us consider two numbers $0<\lambda \leq \Lambda$ and define the Pucci maximal operator as

$$
\mathcal{M}_{\lambda, \Lambda}^{+}(M)=\sup _{a \in[\lambda, \Lambda]^{N}} \sum_{i=1}^{N} a_{i} \lambda_{i}(M),
$$

where $M$ is a symmetric matrix and

$$
\lambda_{1}(M) \leq \lambda_{2}(M) \leq \ldots \leq \lambda_{N}(M)
$$

are the ordered eigenvalues of $M$. When applied to a twice differentiable function $u, \mathcal{M}_{\lambda, \Lambda}^{+}\left(D^{2} u\right)$ is a differential operator that generalizes the Laplacian; actually, it becomes the Laplacian when $\lambda=\Lambda=1$. Associated to $\mathcal{M}_{\lambda, \Lambda}^{+}$there is the dimension-like number

$$
N_{\lambda, \Lambda}^{+}=\frac{\lambda}{\Lambda}(N-1)+1,
$$

whose role in critical exponents for equations such as (1.3) and (1.5) was recently disclosed. Labutin proved in [18] a result similar to Theorem 1.1] where the Laplacian in (1.3) is replaced by the Pucci operator and the critical exponent (1.2) by

$$
p_{*}(\lambda, \Lambda)=\frac{N_{\lambda, \Lambda}^{+}}{N_{\lambda, \Lambda}^{+}-2} .
$$

On the other hand, it was proved by Cutri and Leoni 9 that a result like Theorem 1.2 holds for the Pucci operator instead of the Laplacian and with a critical exponent such as (1.8).

In view of Theorem 1.1, Theorem 1.2 and its extensions to the Pucci maximal operator discussed above, it is natural to ask for a larger class of operators so that these two theorems remain valid. In this article we find a much larger class of operators for which a fundamental solution can be constructed once an appropriate notion of dimension is defined. With these ingredients we can prove theorems generalizing Theorem 1.1 and Theorem 1.2. 
As mentioned above, the main feature of the operators we are considering in this article is that they have an associated dimension number with which we define a fundamental solution. Given a closed, convex, bounded subset $\mathcal{C}$ of $R_{+}^{N}$, we define, for every symmetric matrix $M$, the operator

$$
\mathcal{M}_{\mathcal{C}}^{+}(M)=\sup _{a \in \mathcal{C}} \sum_{i=1}^{N} a_{i} \lambda_{i}(M) \text {. }
$$

Associated to such an operator, in Section 2 we define a dimension-like number $N_{\infty}^{+}=N_{\infty}^{+}(\mathcal{C})$. Now we can write our main theorems, which are extensions of the corresponding results of Brézis and Véron [4] and Labutin [18], and Gidas [13] and Cutri and Leoni [9], respectively.

Theorem 1.3. If $\mathcal{C}$ is a closed, convex, bounded subset of $\mathbb{R}_{+}^{N}$ and $N_{\infty}^{+}>2$, then the singularities of a solution to the equation

$$
\mathcal{M}_{\mathcal{C}}^{+}\left(D^{2} u\right)-|u|^{p-1} u=0 \quad \text { in } \quad B(0, R) \backslash\{0\}
$$

are removable if and only if

$$
p \geq \frac{N_{\infty}^{+}}{N_{\infty}^{+}-2} .
$$

Theorem 1.4. If $\mathcal{C}$ is a closed, convex, bounded subset of $\mathbb{R}_{+}^{N}, N_{\infty}^{+}>2$ and $p>1$, then there are no nontrivial supersolutions for

$$
\mathcal{M}_{\mathcal{C}}^{+}\left(D^{2} u\right)+u^{p}=0, \quad u \geq 0, \quad \text { in } \mathbb{R}^{N}
$$

if and only if

$$
p \leq \frac{N_{\infty}^{+}}{N_{\infty}^{+}-2}
$$

We will see below that the operator $\mathcal{M}_{\mathcal{C}}^{+}$is not necessarily convex and so full regularity theory for (1.9) and (1.11) is not available. Consequently we consider solutions (or supersolutions) in the viscosity sense. Theorem 1.3 and Theorem 1.4 may also be stated and proved for the minimal operators $\mathcal{M}_{\mathcal{C}}^{-}$, which are defined as $\mathcal{M}_{\mathcal{C}}^{+}$, but with the infimum instead of the supremum.

At this point we would like to discuss the relation between the results obtained in this article and those recently obtained by the authors in 12 . If we consider the existence of nonnegative solutions (not supersolutions) to (1.11), then the range of $p$ for nonexistence increases. In particular, if we consider the Laplacian operator, then it is well known that the range for nonexistence is determined by the Sobolev critical exponent

$$
p^{*}=\frac{N+2}{N-2},
$$

as has been proved by Caffarelli, Gidas and Spruck in [6] and Chen and Li [7]. See also Gidas and Spruck [14] and Serrin and Zou [24]. In view of the role of $N_{\infty}^{+}$in Theorem 1.3 and Theorem 1.4 one may think that the quotient $\left(N_{\infty}^{+}+2\right) /\left(N_{\infty}^{+}-2\right)$ would play the role of the Sobolev critical exponent; however this is not the case. In fact, we proved in 12 that there is a critical number $p^{*}$ satisfying

$$
\frac{N_{\infty}^{+}}{N_{\infty}^{+}-2}<p^{*}<\frac{N_{\infty}^{+}+2}{N_{\infty}^{+}-2}
$$


which separates the existence and nonexistence range for $p>1$, in the nontrivial case. In [12] we gave the definition of the dimension number $N_{\infty}^{+}$in a slightly different but equivalent manner. We also mention the earlier article [11, where we obtained such results for the Pucci operators.

We devote the rest of this article to proving Theorems 1.3 and 1.4. In Section 2 we define in a precise manner the class of the maximal and minimal operators we consider in the rest of the article. We see the relation between our class and other natural classes of operators. Then we define the dimension number $N_{\infty}^{+}$and we find fundamental solutions for the maximal (and minimal) operators. In Section 3 we provide a proof of Theorem 1.3. Following the basic approach of [18 we obtained the removability property using a series of estimates. Here we observe that our operator is not convex, so only $C^{1, \alpha}$ regularity is available. In Section 4, we provide a proof of Theorem 1.4; there we use ideas of [9] . In the Appendix we give a proof of a version of the Strong Maximum Principle, which suits our needs.

\section{About extremal operators, Dimension And Fundamental SOlutions}

In this section we discuss in more detail the class of operators we consider. Let $\mathcal{C}$ be a closed, convex, bounded subset of $\mathbb{R}_{+}^{N}$. We denote by $S^{N}$ the set of all $N \times N$ symmetric matrices and for $M \in S^{N}$ we let

$$
\lambda_{1}(M) \leq \lambda_{2}(M) \leq \ldots \leq \lambda_{N}(M)
$$

be the ordered eigenvalues of $M$. Then we define the extremal operators

$$
\mathcal{M}_{\mathcal{C}}^{+}(M)=\sup _{a \in \mathcal{C}} \sum_{i=1}^{N} a_{i} \lambda_{i}(M) \quad \text { and } \quad \mathcal{M}_{\mathcal{C}}^{-}(M)=\inf _{a \in \mathcal{C}} \sum_{i=1}^{N} a_{i} \lambda_{i}(M) .
$$

We say that the set $\mathcal{C}$ is symmetric if

$$
a=\left(a_{1}, a_{2}, \ldots, a_{N}\right) \in \mathcal{C} \quad \text { if and only if } a=\left(a_{\pi(1)}, a_{\pi(2)}, \ldots, a_{\pi(N)}\right) \in \mathcal{C},
$$

for all permutations $\pi$. The Pucci operators correspond to the class of operators, where the set $\mathcal{C}$ is symmetric. For the operators $\mathcal{M}_{\lambda, \Lambda}^{+}$and $\mathcal{M}_{\lambda, \Lambda}^{-}$we define $\mathcal{C}=$ $[\lambda, \Lambda]^{N}$, while for the second class of Pucci operators, we consider $c \in[\lambda, \Lambda]$ and $\mathcal{C}=\left\{a \in[\lambda, \Lambda]^{N} / \sum_{i=1}^{N} a_{i}=c N\right\}$. See [20] and [21].

The class of operators defined above includes operators constructed upon a general set of symmetric matrices. More precisely, let $\mathcal{A} \subset S^{N}$ be such that

$(\mathcal{A} 1)$ There exist numbers $0<\lambda \leq \Lambda$ such that

$$
\lambda I \leq A \leq \Lambda I, \quad \forall A \in \mathcal{A},
$$

$(\mathcal{A} 2) A \in \mathcal{A}$ if and only if $P^{t} A P \in \mathcal{A}$, for all orthogonal matrices $P$.

For such a class of matrices $\mathcal{A}$ we define the extremal operators

$$
\mathcal{P}_{\mathcal{A}}^{+}(M)=\sup _{A \in \mathcal{A}} \operatorname{tr}(A M) \quad \text { and } \quad \mathcal{P}_{\mathcal{A}}^{-}(M)=\inf _{A \in \mathcal{A}} \operatorname{tr}(A M) .
$$

The following lemma connects these two classes of operators.

Lemma 2.1. For every set of symmetric matrices $\mathcal{A}$ satisfying $(\mathcal{A} 1)$ and $(\mathcal{A} 2)$ there exists a convex, bounded, symmetric set $\mathcal{C}$ such that

$$
\mathcal{M}_{\mathcal{C}}^{+}=\mathcal{P}_{\mathcal{A}}^{+} \quad \text { and } \quad \mathcal{M}_{\mathcal{C}}^{-}=\mathcal{P}_{\mathcal{A}}^{-} .
$$


Proof. Given a set $\mathcal{A}$ satisfying $(\mathcal{A} 1)$ and $(\mathcal{A} 2)$, we define the set $\mathcal{C}$ in the following way:

$$
\mathcal{C}=\left\{\left(a_{11}, a_{22}, \ldots, a_{n n}\right) / A=\left(a_{i j}\right) \in \mathcal{A}\right\} .
$$

Then we observe that

$$
\begin{aligned}
\mathcal{P}_{\mathcal{A}}^{+}(M) & =\sup _{A \in \mathcal{A}} \operatorname{tr}(A M) \\
& =\sup _{A \in \mathcal{A}} \operatorname{tr}\left(P^{t} A P D\right),
\end{aligned}
$$

where $M=P D P^{t}$, with $D$ diagonal and $P$ orthogonal. Since $\mathcal{A}=P^{t} \mathcal{A} P$ we see then that

$$
\mathcal{P}_{\mathcal{A}}^{+}(M)=\sup _{A \in \mathcal{A}} \operatorname{tr}(A D)=\sup _{a \in \mathcal{C}} \sum_{i=1}^{N} a_{i} \lambda_{i}(M) .
$$

We observe that the set $\mathcal{C}$ is symmetric and that we can always consider the convex envelope of $\mathcal{C}$, without changing the optimal value.

Still we want to discuss the relation between a class of fully nonlinear operators and the classes defined above. Let $F: S^{N} \rightarrow \mathbb{R}$ be a function satisfying

$(\mathcal{F} 1) F$ is convex and positively homogeneous of degree 1.

$(\mathcal{F} 2)$ There exist numbers $0<\lambda \leq \Lambda$ such that

$$
\mathcal{M}_{\lambda, \Lambda}^{-}(M) \leq F(M) \leq \mathcal{M}_{\lambda, \Lambda}^{+}(M)
$$

for all matrices $M \in S^{N}$.

$(\mathcal{F} 3) F(A)=F\left(P^{t} A P\right)$, for all orthogonal matrices $P$.

We have the following representation lemma for the function $F$ satisfying the three properties just given above.

Lemma 2.2. If $F$ is a function satisfying $(\mathcal{F} 1)-(\mathcal{F} 3)$, then there exists a set $\mathcal{A} \subset S^{N}$ satisfying $(\mathcal{A} 1)-(\mathcal{A} 2)$ such that

$$
F(M)=\mathcal{P}_{\mathcal{A}}^{+}(M) \quad \forall M \in S^{N}
$$

Proof. Let

$$
\mathcal{A}=\left\{A \in S^{N} / \operatorname{tr}(A B) \leq F(B), \forall B \in S^{N}\right\} .
$$

We see that $\mathcal{A}$ satisfies $(\mathcal{A} 1)-(\mathcal{A} 2)$ thanks to $(\mathcal{F} 1)-(\mathcal{F} 2)$. Then, since $F$ is convex, we also have

$$
F(M)=\sup \{\operatorname{tr}(A M) / A \in \mathcal{A}\} .
$$

We observe that the homogeneity of $F$ implies that in recovering $F$ from affine functions, we only need linear functions.

Related to property $(\mathcal{F} 2)$ and the convexity of $F$ we have the property called uniform ellipticity which says: $F$ is uniformly elliptic if

$$
\mathcal{M}_{\lambda, \Lambda}^{-}(B) \leq F(M+B)-F(M) \leq \mathcal{M}_{\lambda, \Lambda}^{+}(B), \quad \forall B \in S^{N} .
$$

It is not hard to see that in (2.3) it is enough to consider only positively semi-defined matrices $B \in S^{N}$.

We can see that the operators $\mathcal{P}_{\mathcal{A}}^{+}$, for any given $\mathcal{A}$ satisfying $(\mathcal{A} 1)-(\mathcal{A} 2)$, are uniformly elliptic and, consequently, so are the operators in the class of $F$ satisfying $(\mathcal{F} 1)-(\mathcal{F} 3)$. We can also prove that the operators $\mathcal{M}_{\mathcal{C}}^{+}$and $\mathcal{M}_{\mathcal{C}}^{-}$are uniformly 
elliptic. In fact, let $0<\lambda \leq \Lambda$ such that $\mathcal{C} \subset[\lambda, \Lambda]^{N}, M$ and $B$ symmetric matrices such that $B \geq 0$, and let $\bar{a} \in \mathcal{C}$ such that

$$
\mathcal{M}_{\mathcal{C}}^{+}(M+B)=\sup _{a \in \mathcal{C}} \sum_{i=1}^{N} a_{i} \lambda_{i}(M+B)=\sum_{i=1}^{N} \bar{a}_{i} \lambda_{i}(M+B) .
$$

Then we have

$$
\mathcal{M}_{\mathcal{C}}^{+}(M+B)-\sum_{i=1}^{N} \bar{a}_{i} \lambda_{i}(M)=\sum_{i=1}^{N} \bar{a}_{i}\left(\lambda_{i}(M+B)-\lambda_{i}(M)\right) \leq \Lambda \operatorname{tr}(B),
$$

from which it follows that

$$
\mathcal{M}_{\mathcal{C}}^{+}(M+B)-\mathcal{M}_{\mathcal{C}}^{+}(M) \leq \Lambda \operatorname{tr}(B)
$$

Proceeding in a similar form we also obtain

$$
\mathcal{M}_{\mathcal{C}}^{+}(M+B)-\mathcal{M}_{\mathcal{C}}^{+}(M) \geq \lambda \operatorname{tr}(B)
$$

and then (2.3) follows. Similarly, we can prove that the operators $\mathcal{M}_{\mathcal{C}}^{-}$are uniformly elliptic. Here and in what follows we denote by $\operatorname{tr}(M)$ the trace of the matrix $M$.

On the other hand, the operators $\mathcal{M}_{\mathcal{C}}^{+}$and $\mathcal{M}_{\mathcal{C}}^{-}$are not convex in general. In order to prove this we provide a simple example. We consider the matrices

$$
M=\left(\begin{array}{cc}
m & 0 \\
0 & 0
\end{array}\right) \quad \text { and } B=\left(\begin{array}{cc}
0 & 0 \\
0 & n
\end{array}\right)
$$

where $m>n>0$. We also consider the set $\mathcal{C}=[1,8] \times[1,2]$. Then we have

$$
\mathcal{M}_{\mathcal{C}}^{+}(M)=2 m, \quad \mathcal{M}_{\mathcal{C}}^{+}(B)=2 n \quad \text { and } \quad \mathcal{M}_{\mathcal{C}}^{+}(M+B)=2 m+8 n,
$$

and so the inequality

$$
\mathcal{M}_{\mathcal{C}}^{+}(M+B) \leq \mathcal{M}_{\mathcal{C}}^{+}(M)+\mathcal{M}_{\mathcal{C}}^{+}(B),
$$

which is a consequence of convexity, does not hold.

We conclude this section by discussing the concept of dimension associated to the operators $\mathcal{M}_{\mathcal{C}}^{+}$and $\mathcal{M}_{\mathcal{C}}^{-}$. Define

$$
c(\alpha)=\max _{a \in \mathcal{C}}-\sum_{i=1}^{N-1} a_{i}+(\alpha-1) a_{N} .
$$

We assume from now on that the set $\mathcal{C}$ is such that $c(2)<0$. We see that the function $c$ is continuous, strictly increasing and it satisfies $c(\alpha)>0$ for large $\alpha$. Thus, there exists a unique number, which we call $N_{\infty}^{+}$, such that $c\left(N_{\infty}^{+}\right)=0$. This number is the dimension associated to $\mathcal{M}_{\mathcal{C}}^{+}$.

If we define

$$
b(\alpha)=\min _{a \in \mathcal{C}}-\sum_{i=1}^{N-1} a_{i}+(\alpha-1) a_{N},
$$

then we see that $b(2) \leq c(2)<0$. Thus, there exists a unique number, which we call $N_{\infty}^{-}$, such that $b\left(N_{\infty}^{-}\right)=0$. This number $N_{\infty}^{-}$is the dimension associated to $\mathcal{M}_{\mathcal{C}}^{-}$. 
Remark 2.1. We observe that $N_{\infty}^{+} \leq N_{\infty}^{-}$. We also see that

$$
N_{\infty}^{+}-1 \leq \frac{\sum_{i=1}^{N-1} a_{i}}{a_{N}}, \quad \forall a \in \mathcal{C}
$$

and

$$
N_{\infty}^{-}-1 \geq \frac{\sum_{i=1}^{N-1} a_{i}}{a_{N}}, \quad \forall a \in \mathcal{C} .
$$

Remark 2.2. In case $c(2) \geq 0$, we can also define dimension-like numbers, but then $N_{\infty}^{+} \leq 2$. Throughout the paper we will always assume that $c(2)<0$ and that $2<N_{\infty}^{+} \leq N_{\infty}^{-}$.

The motivation for defining the dimension numbers comes from the definition of fundamental solutions. When $N_{\infty}^{+}>2$ we define the fundamental solutions of $\mathcal{M}_{\mathcal{C}}^{+}$ and $\mathcal{M}_{\mathcal{C}}^{-}$, respectively, as

$$
\phi^{+}(x)=\frac{1}{|x|^{N_{\infty}^{+}-2}} \quad \text { and } \quad \phi^{-}(x)=\frac{1}{|x|^{N_{\infty}^{-}-2}},
$$

and see that

$$
\mathcal{M}_{\mathcal{C}}^{+}\left(D^{2} \phi^{+}\right)=0 \quad \text { and } \quad \mathcal{M}_{\mathcal{C}}^{-}\left(D^{2} \phi^{-}\right)=0
$$

We also see that

$$
\mathcal{M}_{\mathcal{C}}^{-}\left(D^{2}\left(-\phi^{+}\right)\right)=0 \quad \text { and } \quad \mathcal{M}_{\mathcal{C}}^{+}\left(D^{2}\left(-\phi^{-}\right)\right)=0 .
$$

See [18, 19] and 9] for further discussion on the fundamental solutions for the Pucci operators $\mathcal{M}_{\lambda, \Lambda}^{+}$and $\mathcal{M}_{\lambda, \Lambda}^{-}$.

\section{Proof of Theorem 1.3}

In this section we provide a proof of Theorem 1.3, which is a consequence of a more general theorem that involves more general nonlinearities. But before going to that, we state a Comparison Principle due to Ishi and Lions [16], which will be used repeatedely in the proof of our results. See also Jensen [17].

Theorem 3.1. Assume $\Omega$ is a bounded domain in $\mathbb{R}^{N}$. Let $F: S^{N} \times \mathbb{R} \rightarrow \mathbb{R}$ be a continuous function satisfying the following two conditions:

(1) There is a positive constant $c_{0}$ such that

$$
F(M+B, t)-F(M, t) \geq c_{0} \operatorname{tr}(B),
$$

for all $M, B \in S^{N}, N \geq 0$ and $t \in \mathbb{R}$.

$$
F(M, t) \leq F(M, s) \quad \forall t \geq s, M \in S^{N} .
$$

If $u, v \in C(\bar{\Omega})$ are the subsolution and the supersolution of

$$
F\left(D^{2} u, u\right)=0 \text { in } \Omega,
$$

respectively, such that $u(x) \leq v(x)$ for all $x \in \partial \Omega$, then $u(x) \leq v(x)$ for all $x \in \Omega$.

Remark 3.1. In Theorem 3.1 we may assume that $F$ depends also on $x$. Specifically we may assume that $F: S^{N} \times \mathbb{R}: \Omega \rightarrow \mathbb{R}$ is a continuous function satisfying $x$ dependent versions of (1) and (2) and also satisfying

$$
|F(M, t, x)-F(M, t, y)| \leq c|x-y|, \quad \forall x, y \in \Omega .
$$


Then the comparison result holds for the equation

$$
F\left(D^{2} u, u, x\right)=0 \quad \text { in } \Omega .
$$

Now we consider a more general version of Theorem 3.1.

Theorem 3.2. Let $F: S^{N} \rightarrow \mathbb{R}$ be a continuous function such that, for some closed, convex, bounded subset $\mathcal{C}$ of $\mathbb{R}_{+}^{N}$,

$$
\mathcal{M}_{\mathcal{C}}^{-}(M) \leq F(M)-F(0) \leq \mathcal{M}_{\mathcal{C}}^{+}(M),
$$

for all $M \in S^{N}$. Assume that $N_{\infty}^{+}>2$ and also that $f: \mathbb{R} \rightarrow \mathbb{R}$ is a continuous function satisfying

$$
\limsup _{t \rightarrow \infty} \frac{f(t)}{t^{N_{\infty}^{+} /\left(N_{\infty}^{+}-2\right)}}<0 \quad \text { and } \quad \liminf _{t \rightarrow-\infty} \frac{f(t)}{|t|^{N_{\infty}^{+} /\left(N_{\infty}^{+}-2\right)}}>0 .
$$

If $u \in C_{l o c}\left(B_{R} \backslash\{0\}\right)$ is a solution of the equation

$$
F\left(D^{2} u\right)+f(u)=0 \quad \text { in } \quad B_{R} \backslash\{0\},
$$

then $u$ can be extended continuously to 0 as a solution of (3.3) in $B_{R}$.

We will prove Theorem 3.2 following the main ideas developed by Labutin in [18. We prove first that $u$ can be defined as a continuous function at the origin.

Lemma 3.1. Under the hypotheses of Theorem 3.2, the function u satisfies

$$
\lim _{x \rightarrow 0} u(x)=u_{0},
$$

for some $u_{0}$ in $\mathbb{R}$.

Proof. We consider $u=u^{+}+u^{-}$, where $u^{+}=\max \{u, 0\}$ and $u^{-}=\min \{u, 0\}$, and we write $p_{*}=N_{\infty}^{+} /\left(N_{\infty}^{+}-2\right)$. We start by proving that there is a constant $\beta$ such that

$$
u^{+}(x) r^{N_{\infty}^{+}-2} \leq \beta, \quad \forall x \in B_{R / 2} \backslash\{0\} .
$$

Using assumptions (3.1), (3.2) and the definition of a viscosity solution, after proper scaling, we find that

$$
\mathcal{M}_{\mathcal{C}}^{+}\left(D^{2} u^{+}\right)-\left(u^{+}\right)^{p_{*}}+b \geq 0, \quad \forall x \in B_{R / 2} \backslash\{0\},
$$

where $b \geq 0$ depends on (3.2). We consider the comparison function

$$
U(x)=\frac{\mu}{\left(\rho^{2}-r^{2}\right)^{N_{\infty}^{+}-2}}+\nu, \quad x \in B_{\rho},
$$

where the constants $\rho, \mu$ and $\nu$ are chosen so that $U$ satisfies

$$
\mathcal{M}_{\mathcal{C}}^{+}\left(D^{2} U\right)-U^{p_{*}}+b \leq 0 \quad \forall x \in B_{\rho},
$$

and $\lim _{r \rightarrow \rho} U(x)=\infty$. See Brézis and Véron [4]. In order to see this, we notice that the ordered eigenvalues of $D^{2} U$ are

$$
\lambda_{1}=\ldots=\lambda_{N-1}=\frac{U^{\prime}}{r}=\frac{2 \mu\left(N_{\infty}^{+}-2\right)}{\left(\rho^{2}-r^{2}\right)^{N_{\infty}^{+}-1}}
$$

and

$$
\lambda_{N}=U^{\prime \prime}=\frac{2 \mu\left(N_{\infty}^{+}-2\right)\left(\rho^{2}+\left(2 N_{\infty}^{+}-3\right) r^{2}\right)}{\left(\rho^{2}-r^{2}\right)^{N_{\infty}^{+}}} .
$$


Considering $x \in B_{\rho}$ we get from here that

$$
\lambda_{1}=\ldots=\lambda_{N-1} \leq \frac{2 \mu \rho^{2}\left(N_{\infty}^{+}-2\right)}{\left(\rho^{2}-r^{2}\right)^{N_{\infty}^{+}}}, \quad \lambda_{N} \leq \frac{4 \mu \rho^{2}\left(N_{\infty}^{+}-2\right)\left(N_{\infty}^{+}-1\right)}{\left(\rho^{2}-r^{2}\right)^{N_{\infty}^{+}}}
$$

and then

$$
\begin{aligned}
\mathcal{M}_{\mathcal{C}}^{+}\left(D^{2} U\right) & =\max _{a \in \mathcal{C}} \sum_{i=1}^{N-1} a_{i} \frac{U^{\prime}}{r}+a_{N} U^{\prime \prime} \\
& \leq 2 \mu \rho^{2} \Lambda \frac{\left(N_{\infty}^{+}-2\right)(N-1)+2\left(N_{\infty}^{+}-2\right)\left(N_{\infty}^{+}-1\right)}{\left(\rho^{2}-r^{2}\right)^{N_{\infty}^{+}}}
\end{aligned}
$$

where the numbers $0<\lambda \leq \Lambda$ are such that $\mathcal{C} \subset[\lambda, \Lambda]^{N}$. On the other hand, we have that

$$
U^{N_{\infty}^{+} /\left(N_{\infty}^{+}-2\right)} \geq \frac{1}{2} \frac{\mu^{N_{\infty}^{+} /\left(N_{\infty}^{+}-2\right)}}{\left(\rho^{2}-r^{2}\right)^{N_{\infty}^{+}}}+\frac{1}{2} \nu^{N_{\infty}^{+} /\left(N_{\infty}^{+}-2\right)}
$$

If we choose

$$
\mu=\left(4 \Lambda \rho^{2}\left\{\left(N_{\infty}^{+}-2\right)(N-1)+2\left(N_{\infty}^{+}-2\right)\left(N_{\infty}^{+}-1\right)\right\}\right)^{\left(N_{\infty}^{+}-2\right) / 2}
$$

and

$$
\nu=(2 b)^{\left(N_{\infty}^{+}-2\right) / N_{\infty}^{+}},
$$

then, from (3.7) and (3.8), we obtain (3.6). Next we consider $x_{0} \in B_{R / 4}$ and $\rho=$ $\left|x_{0}\right| / 2$. We use Theorem 3.1 in order to compare $u^{+}$and $U\left(x-x_{0}\right)$ in $B\left(x_{0},\left|x_{0}\right| / 2\right)$ and we obtain $u^{+}\left(x_{0}\right) \leq U(0)$. From here (3.4) follows.

Next we prove an improved version of (3.4), that is,

$$
\lim _{x \rightarrow 0} u^{+}(x) r^{N_{\infty}^{+}-2}=0 .
$$

We assume, for contradiction, that there is a constant $K>0$ such that

$$
\limsup _{x \rightarrow 0} u^{+}(x) r^{N_{\infty}^{+}-2}=K .
$$

For comparison, we define the function

$$
v(x)=\frac{K}{r^{N_{\infty}^{+}-2}}+\max _{\partial B_{R / 2}} u^{+}+b+1, \quad x \in B_{R / 2} \backslash\{0\}
$$

and we notice that

$$
\mathcal{M}_{\mathcal{C}}^{+}\left(D^{2} v\right)-v^{p_{*}}+b \leq 0 \quad \text { in } B_{R / 2} \backslash\{0\} .
$$

Using the comparison Theorem 3.1 we obtain that

$$
u^{+} \leq v \quad \text { in } B_{R / 2} \backslash\{0\} .
$$

This requires some discussion: we replace $K$ by $K+\varepsilon$ in the definition of $v$ above and we apply the comparison theorem in the domain $B_{R / 2} \backslash B_{\rho_{\varepsilon}}$, for some $\rho_{\varepsilon}>0$, such that $\rho_{\varepsilon} \rightarrow 0$ as $\varepsilon \rightarrow 0$. Taking the limit we obtain the result.

Next we obtain a sequence of points $\left\{x_{i}\right\}$ satisfying $x_{i} \rightarrow 0$ and

$$
\lim _{i \rightarrow \infty} \frac{u^{+}\left(x_{i}\right)}{v\left(x_{i}\right)}=1 .
$$


Defining $\delta_{i}=\left|x_{i}\right|$ and $\Omega_{i}=B_{2 \delta_{i}} \backslash \bar{B}_{\delta_{i} / 2}$ we consider the boundary value problem

$$
\begin{aligned}
\mathcal{M}_{\mathcal{C}}^{+}\left(D^{2} w_{i}\right)-\left|w_{i}\right|^{p_{*}-1} w_{i}+b & =0 \text { in } \Omega_{i}, \\
w_{i} & =u^{+} \text {on } \partial \Omega_{i} .
\end{aligned}
$$

We see that $u^{+}$is a $C\left(\bar{\Omega}_{i}\right)$ viscosity subsolution of equation (3.11)-(3.12). On the other hand, the function $v$ is a $C\left(\bar{\Omega}_{i}\right)$ viscosity supersolution of (3.11)-(3.12). The operator $F(M, t)=\mathcal{M}_{\mathcal{C}}^{+}(M)-|t|^{p^{*}-1} t+b$ satisfies the properties of Theorem 3.1 so that by Perron's method we can construct a continuous viscosity solution $w_{i}$ to (3.11)-(3.12), which additionally satisfies

$$
u^{+} \leq w_{i} \leq v \quad \text { in } \quad \Omega_{i} .
$$

Now we use a rescaling argument as in [18. Consider $\Omega=B_{2} \backslash \bar{B}_{1 / 2}$ and for every $i$ define

$$
v_{\delta_{i}}(x)=\delta_{i}^{N_{\infty}^{+}-2} v\left(\delta_{i} x\right) \quad \text { and } \quad w_{\delta_{i}}(x)=\delta_{i}^{N_{\infty}^{+}-2} w_{i}\left(\delta_{i} x\right), \quad x \in \Omega .
$$

By definition, it is clear that $v_{\delta_{i}}$ converges uniformly to $K r^{-N_{\infty}^{+}+2}$ over $\Omega$. We also see that $w_{\delta_{i}}$ is uniformly bounded in $\Omega$ and satisfies

$$
\mathcal{M}_{\mathcal{C}}^{+}\left(D^{2} w_{\delta_{i}}\right)-\left|w_{\delta_{i}}\right|^{p_{*}-1} w_{\delta_{i}}+\delta_{i}^{N_{\infty}^{+}} b=0 \text { in } \Omega .
$$

Here we may use the $C^{\alpha}$ regularity estimates, see for example Cabré and Caffarelli [5], to obtain that $\left\|w_{\delta_{i}}\right\|_{C^{\alpha}(\bar{\Omega})}$ is bounded, for $\alpha \in(0,1)$. This implies that, up to a subsequence, $\left\{w_{\delta_{i}}\right\}$ converges uniformly to a $w \in C(\bar{\Omega})$ satisfying

$$
\mathcal{M}_{\mathcal{C}}^{+}\left(D^{2} w\right)-|w|^{p_{*}-1} w=0 \quad \text { in } \Omega
$$

and $0 \leq w(x) \leq K r^{-N_{\infty}^{+}+2}$ in $\Omega$. Moreover, there exists $x_{0} \in \Omega$ such that $w\left(x_{0}\right)=$ $K\left|x_{0}\right|^{-N_{\infty}^{+}+2}$, so that, by the Strong Maximum Principle (see Appendix) $w(x)=$ $K r^{-N_{\infty}^{+}+2}+C$ in $\Omega$, which implies $K=0$, a contradiction.

Once we have (3.9) we may compare again $u^{+}$with

$$
v_{\varepsilon}(x)=\frac{\varepsilon}{r^{N_{\infty}^{+}-2}}+\max _{\partial B_{R / 2}} u^{+}+b+1, \quad x \in B_{R / 2} \backslash\{0\},
$$

and take $\varepsilon \rightarrow 0$, to obtain $u^{+} \in L^{\infty}\left(B_{R / 2}\right)$.

Using similar arguments we obtain $u^{-} \in L^{\infty}\left(B_{R / 2}\right)$. Here we start the argument by proving the existence of $\beta \geq 0$ such that, instead of (3.4),

$$
-\beta \leq u^{-}(x) r^{N_{\infty}^{+}-2} .
$$

We notice that $-r^{-N_{\infty}^{+}+2}$ is a fundamental solution for $\mathcal{M}_{\mathcal{C}}^{-}$.

We can conclude then that $u \in L^{\infty}\left(B_{R / 2}\right)$. To complete the proof we observe that for some $0<\lambda \leq \Lambda$ such that $\mathcal{C} \subset[\lambda, \Lambda]^{N}$ we have

$$
\mathcal{M}_{\lambda, \Lambda}^{+}\left(D^{2} u\right) \geq-|g(x)| \text { and } \mathcal{M}_{\lambda, \Lambda}^{-}\left(D^{2} u\right) \leq|g(x)| \text { in } \Omega,
$$

where $g(x)=f(u(x))-F(0)$ is continuous. Consequently we may use the KrylovSafonov-Harnarck inequality and the Alexandrof-Bakelman-Pucci estimate as in [18] to obtain the continuity of $u$ at 0 , by defining $u(0)$ properly.

Proof of Theorem 3.2, Once we have the continuity of $u$ at the origin, we just need to prove that the function $u$ is a viscosity solution in 0. For this purpose we may follow step-by-step the arguments in [18, only changing the Pucci operator by $\mathcal{M}_{\mathcal{C}}^{+}$ and using the function $r^{-N_{\infty}^{+}+2}$ instead of $E^{+}$. 
Finally, we complete the proof of Theorem 1.3 by exhibiting a solution $u$ of (1.9), which is singular at 0 , for every $p \in\left(1, p_{*}\right)$.

Proof of Theorem 1.3. Assume $N_{\infty}^{+}>2$ and that $p \in\left(1, p_{*}\right)$. We consider the function

$$
u(x)=a|x|^{-2 /(p-1)},
$$

where $a$ is chosen below. We observe that $\alpha:=2 p /(p-1)>p_{*}>2$, so that

$$
\mathcal{M}_{\mathcal{C}}^{+}\left(D^{2} u(x)\right)=r^{-2 p /(p-1)} a(\alpha-2) c(\alpha),
$$

where $c(\alpha)>0$. Since $u^{p}=a^{p} r^{-2 p /(p-1)}$, we may choose $a$ so that

$$
a^{p-1}=(\alpha-2) c(\alpha)
$$

and then we obtain a solution of

$$
\mathcal{M}_{\mathcal{C}}^{+}\left(D^{2} u\right)-u^{p}=0
$$

with a singularity at the origin. Similarly we can construct a singular solution for the operator $\mathcal{M}_{\mathcal{C}}^{-}$.

\section{Proof of Theorem 1.4}

We devote this section to prove Theorem 1.4 a Liouville-type theorem for supersolutions for the maximal operators $\mathcal{M}_{\mathcal{C}}^{+}$.

A version of Theorem 1.4 can be proved for the minimal operator and $\mathcal{M}_{\mathcal{C}}^{-}$, replacing $N_{\infty}^{+}$by $N_{\infty}^{-}$. We notice that this version of Theorem 1.4 for $\mathcal{M}_{\mathcal{C}}^{-}$implies a Liouville-type theorem for super-solutions of

$$
F\left(D^{2} u\right)+u^{p}=0, \quad u \geq 0, \quad \text { in } \mathbb{R}^{N},
$$

if $\mathcal{M}_{\mathcal{C}}^{-}(M) \leq F(M)$ for any symmetric matrix $M$ and $p \leq N_{\infty}^{-} /\left(N_{\infty}^{-}-2\right)$.

We start our discussion with a version of the Hadamard Three Spheres Theorem for the extremal operators. Given $u \in C\left(B_{R}\right)$, let us define $m(r)=\min _{|x| \leq r} u(x)$, for $0 \leq r<R$.

Theorem 4.1. Assume that $N_{\infty}^{+}>2$. If $u \in C\left(B_{R}\right)$ is a positive viscosity solution of

$$
\mathcal{M}_{\mathcal{C}}^{+}\left(D^{2} u\right) \leq 0, \quad \text { in } \quad B_{R}
$$

then for any $0<r_{1}<r<r_{2}<R$ we have

$$
m(r) \geq \frac{m\left(r_{1}\right)\left(r^{2-N_{\infty}^{+}}-r_{2}^{2-N_{\infty}^{+}}\right)+m\left(r_{2}\right)\left(r_{1}^{2-N_{\infty}^{+}}-r^{2-N_{\infty}^{+}}\right)}{r_{1}^{2-N_{\infty}^{+}}-r_{2}^{2-N_{\infty}^{+}}} .
$$

A similar result holds for $\mathcal{M}_{\mathcal{C}}^{-}$, replacing $N_{\infty}^{+}$by $N_{\infty}^{-}$.

Proof. Let us define $\phi(x)=C_{1}|x|^{2-N_{\infty}^{+}}+C_{2}$, where $C_{1}$ and $C_{2}$ are such that $\phi(x)=m\left(r_{1}\right)$ on $\partial B_{r_{1}}$ and $\phi(x)=m\left(r_{2}\right)$ on $\partial B_{r_{2}}$. Since $\mathcal{M}_{\mathcal{C}}^{+}\left(D^{2} \phi\right)=0$ in $B_{r_{2}} \backslash B_{r_{1}}$, using comparison Theorem 3.1 we get $u(x) \geq \phi(x)$ in $B_{r_{2}} \backslash B_{r_{1}}$ which implies the result.

Remark 4.1. By the Strong Maximum Principle, Theorem A.1 in the Appendix, given $u \in C\left(B_{R}\right)$ satisfying (4.1), we have

$$
m(r)=\min _{|x| \leq r} u(x)=\min _{|x|=r} u(x) .
$$


Corollary 4.1. If $u \in C\left(\mathbb{R}^{N}\right)$ is a positive viscosity solution of

$$
\mathcal{M}_{\mathcal{C}}^{+}\left(D^{2} u\right) \leq 0, \quad \text { in } \quad \mathbb{R}^{N}
$$

then the function $r_{\infty}^{N_{\infty}^{+}-2} m(r)$ is increasing.

A similar result holds for $\mathcal{M}_{\mathcal{C}}^{-}$, replacing $N_{\infty}^{+}$by $N_{\infty}^{-}$.

Proof. First, we observe that $u(x)>0$ everywhere, by the Strong Maximum Principle, Theorem A.1 in the Appendix. Since the inequality (4.2) holds for all $r_{2}$, we may take $r_{2} \rightarrow \infty$. Then, using the positivity of $m(r)$ and that $N_{\infty}^{+}>2$, we conclude the proof.

Proof of Theorem 1.4. Assume, for contradiction, that $u$ is a nontrivial super-solution to equation (1.11). For given $r_{1}>0$ fixed, we consider the function

$$
g(r)=m\left(r_{1} / 2\right)\left(1-\left[\left(r-r_{1} / 2\right)^{+}\right]^{3} /\left(r_{1} / 2\right)^{3}\right),
$$

as in [9]. If we define $\phi(x)=g(|x|)$, then the minimum of $u-\phi$ in $\mathbb{R}^{N}$ is nonpositive and it is achieved at a point $\bar{x}=x\left(r_{1}\right)$, such that $r_{1} / 2 \leq|\bar{x}|<r_{1}$. Thus we can use $\phi$ as a test function of (1.11) at $\bar{x}$ and get

$$
\mathcal{M}_{\mathcal{C}}^{+}\left(D^{2} \phi(\bar{x})\right)+u(\bar{x})^{p} \leq 0 .
$$

Then

$$
u(\bar{x})^{p} \leq \frac{3 m\left(r_{1} / 2\right)}{\left(r_{1} / 2\right)^{3}}\left[a_{N}+\hat{a} \frac{\left(|\bar{x}|-r_{1} / 2\right)^{+}}{|\bar{x}|}\right]\left(|\bar{x}|-r_{1} / 2\right)^{+},
$$

where $a=\left(a_{1}, \ldots, a_{N}\right)$ is some point in $\mathcal{C}$ and $\hat{a}:=\sum_{i=1}^{N-1} a_{i}$. If $|\bar{x}|=r_{1} / 2$, then we have $u(|\bar{x}|)=0$, which is impossible. Thus, we necessarily have $r_{1} / 2<\bar{x}<r_{1}$. Since $m\left(r_{1}\right) \leq u(\bar{x}) \leq m\left(r_{1} / 2\right)$, we get

$$
m\left(r_{1}\right) \leq C \frac{m\left(r_{1} / 2\right)^{1 / p}}{\left(r_{1} / 2\right)^{2 / p}}
$$

for some positive $C$. Now we use Corollary 4.1 to obtain

$$
m\left(r_{1}\right) \leq C \frac{1}{\left(r_{1}\right)^{2 /(p-1)}},
$$

where $C$ is possibly different, but independent of $r_{1}$. Hence

$$
r_{1}^{N_{\infty}^{+}-2} m\left(r_{1}\right) \leq C \frac{1}{\left(r_{1}\right)^{2 /(p-1)-N_{\infty}^{+}+2}} .
$$

If $p<N_{\infty}^{+} /\left(N_{\infty}^{+}-2\right)$, then the increasing function $r_{1}^{N_{\infty}^{+}-2} m\left(r_{1}\right)$ goes to 0 as $r_{1} \rightarrow \infty$, providing a contradiction.

In case $p=N_{\infty}^{+} /\left(N_{\infty}^{+}-2\right)$ we need an extra logarithmic lower bound. Define, for fixed $0<r_{1}<r_{2}$,

$$
h(r)=c_{1} \frac{\log (1+r)}{r^{N_{\infty}^{+}-2}}+c_{2},
$$

where $c_{1}>0$ and $c_{2} \in \mathbb{R}$ are such that $h\left(r_{1}\right) \leq m\left(r_{1}\right)$ and $h\left(r_{2}\right)=m\left(r_{2}\right)$. We may choose $r_{1}$ large enough so that $h^{\prime \prime}(r)>0$ and $h^{\prime}(r)<0$, for $r>r_{1}$. Let $w(x)=h(|x|)$. Then

$$
\mathcal{M}_{\mathcal{C}}^{+}\left(D^{2} w(x)\right)=a_{N} h^{\prime \prime}(r)+\hat{a} \frac{h^{\prime}(r)}{r}, \quad r_{1} \leq r \leq r_{2},
$$


for some $a \in \mathcal{C}$. Then, using the maximal character of the operator, we obtain

$$
\mathcal{M}_{\mathcal{C}}^{+}\left(D^{2} w(x)\right) \geq a_{N}^{\infty}\left(h^{\prime \prime}(r)+\left(N_{\infty}^{+}-1\right) \frac{h^{\prime}(r)}{r}\right) \geq-C \frac{c_{1}}{|x|^{N_{\infty}^{+}}}
$$

where $a^{\infty}=\left(a_{1}^{\infty}, \ldots, a_{N}^{\infty}\right) \in \mathcal{C}$ is such that $\hat{a}^{\infty} / a_{N}^{\infty}=N_{\infty}^{+}-1$, and $C$ is a positive constant.

On the other hand, using Corollary 4.1, we have

$$
u(x) \geq \frac{m\left(r_{1}\right) r_{1}^{N_{\infty}^{+}-2}}{|x|^{N_{\infty}^{+}-2}}
$$

for $|x| \geq r_{1}$, and then from equation (1.11) we obtain

$$
\mathcal{M}_{\mathcal{C}}^{+}\left(D^{2} u(x)\right) \leq-\frac{C_{1}}{|x|^{N_{\infty}^{+}}}
$$

for some positive $C_{1}$. Therefore, we can use comparison Theorem $\left[3.1\right.$, choosing $c_{1}$ smaller if necessary, to conclude that $w(x) \leq u(x)$ in $B_{r_{2}} \backslash B_{r_{1}}$. Letting $r_{2} \rightarrow \infty$ we finally conclude that $c_{2}=0$ and

$$
u(x) \geq \frac{C \log (1+|x|)}{|x|^{N_{\infty}^{+}-2}},
$$

for $|x|$ large and positive $C$. This is a contradiction with the previous estimate (4.3).

We notice that the arguments in the case of $\mathcal{M}_{\mathcal{C}}^{-}$are similar, just replacing $N_{\infty}^{+}$ by $N_{\infty}^{-}$, except for those leading to (4.4). Here, we consider Remark 2.1 to get

from which

$$
a_{N} h^{\prime \prime}(r)+\hat{a} \frac{h^{\prime}(r)}{r} \geq a_{N}\left(h^{\prime \prime}+\left(N_{\infty}^{-}-1\right) \frac{h^{\prime}(r)}{r}\right)
$$

$$
\mathcal{M}_{\mathcal{C}}^{-}\left(D^{2} w(x)\right) \geq-C \frac{c_{1}}{|x|^{N_{\infty}^{-}}}
$$

follows.

To complete the proof we will construct a supersolution of (1.11), when $p>$ $N_{\infty}^{+} /\left(N_{\infty}^{+}-2\right)$. We let $q$ be such that

$$
\frac{1}{p-1}<q<\frac{N_{\infty}^{+}-2}{2}
$$

and we define $u(r)=C_{q}\left(1+r^{2}\right)^{-q}$. We prove that $u$ is a radial supersolution, for an appropriate choice of $C_{q}$. We first observe that $u^{\prime \prime}(r) \geq u^{\prime}(r) / r$ for all $r>0$. Then for the function $v(x)=u(|x|)$ we have

$$
\mathcal{M}_{\mathcal{C}}^{+}\left(D^{2} v(x)\right)=a_{N}\left(u^{\prime \prime}(r)+\frac{\hat{a}}{a_{N}} \frac{u^{\prime}(r)}{r}\right)
$$

for some $a \in \mathcal{C}$. Next we notice that $u^{\prime \prime}(r)+\frac{\hat{a}}{a_{N}} u^{\prime}(r) / r \leq 0$ for all $r$. In fact, using Remark 2.1 and after some calculations, we have

$$
u^{\prime \prime}(r)+\frac{\hat{a}}{a_{N}} u^{\prime}(r) / r \leq u^{\prime \prime}(r)+\left(N_{\infty}^{+}-1\right) u^{\prime}(r) / r \leq-\frac{2 \bar{C}_{q}\left(N_{\infty}^{+}-2(q+1)\right)}{\left(1+r^{2}\right)^{q+1}} \leq 0 .
$$

Next we define $\bar{a}_{m}=\min _{a \in \mathcal{C}} a_{N}$ and we obtain

$$
\mathcal{M}_{\mathcal{C}}^{+}\left(D^{2} v(x)\right) \leq \bar{a}_{m}\left(u^{\prime \prime}(r)+\left(N_{\infty}^{+}-1\right) \frac{u^{\prime}(r)}{r}\right)
$$


since $\frac{\hat{a}}{a_{N}} \geq N_{\infty}^{+}-1$ and $u^{\prime} \leq 0$. Thus

$$
\mathcal{M}_{\mathcal{C}}^{+}\left(D^{2} v\right)+v^{p} \leq-\frac{2 \bar{a}_{m} C_{q}\left(N_{\infty}^{+}-2(q+1)\right)}{\left(1+r^{2}\right)^{q+1}}+\frac{C_{q}^{p}}{\left(1+r^{2}\right)^{p q}} .
$$

Therefore by the definition of $q$, we may choose $C_{q}$ small enough such that the right hand side is always nonpositive. Thus, we have obtained

$$
\mathcal{M}_{\mathcal{C}}^{+}\left(D^{2} v(x)\right)+v^{p}(x) \leq 0 \quad \text { in } \quad \mathbb{R}^{N} .
$$

For the operator $\mathcal{M}_{\mathcal{C}}^{-}$and $N_{\infty}^{-}$the last argument follows just from the minimal character of the operator.

\section{Appendix: The Strong Maximum Principle}

Here we present a form of the Strong Maximum Principle, which is needed in our arguments. Its proof is essentially given by Birindelli and Demengel [3], but we present it here for completeness.

Theorem A.1. Let $\Omega$ be a domain, $c \geq 0, p \geq 1$ and $u, v$ continuous functions in $\Omega$. Assume $u$ is a subsolution of

$$
\mathcal{M}_{\mathcal{C}}^{+}\left(D^{2} u\right)-c|u|^{p-1} u=0,
$$

and that $v$ is a supersolution of (4.6). Moreover, assume that $v(x) \geq u(x)$, for all $x \in \Omega$, and that for some $x_{0} \in \Omega$ it follows that $u\left(x_{0}\right)=v\left(x_{0}\right)$. If either $u$ or $v$ is of class $C^{2}(\Omega)$, then $u(x)=v(x)$ for all $x \in \Omega$.

Proof. Let us assume, without loss of generality, that $u$ is of class $C^{2}(\Omega)$. If $u \neq v$, then there is a point $x_{1} \in \Omega$ and $R>0$ such that $B\left(x_{1}, 3 R / 2\right) \subset \Omega,\left|x_{0}-x_{1}\right|=R$ and $x_{0}$ is the only point in $\bar{B}\left(x_{1}, R\right)$ such that $u\left(x_{0}\right)=v\left(x_{0}\right)$. We assume from now on that $x_{1}=0$, by performing a proper translation.

Since $\mathcal{M}_{\mathcal{C}}^{+}$is uniformly elliptic, say with ellipticity constants $0<\lambda \leq \Lambda$, and since $u$ is of class $C^{2}(\Omega)$, we can see that $w=v-u$ is a viscosity supersolution of

$$
\mathcal{M}_{\lambda, \Lambda}^{+}\left(D^{2} w\right)-c(x) w=0
$$

where $c(x)=c\left(v^{p}(x)-u^{p}(x)\right) /(u(x)-v(x))$ if $u(x) \neq v(x)$ and $c(x)=1$ whenever $u(x)=v(x)$. We observe that $c(x)$ is bounded and that $c(x) \geq 0$ for all $x \in \Omega$.

Let $w_{1}=\inf _{|x|=R / 2} w(x)$. By continuity of $w$ we see that $w_{1}>0$. Next we construct an appropriate subsolution in the annulus $A_{R}=\{x / R / 2<|x|<3 R / 2\}$ by considering $\phi(x)=a e^{-\alpha r}$, with $r=|x|$ and $\alpha$ a constant chosen so that

$$
\mathcal{M}_{\lambda, \Lambda}^{+}\left(D^{2} \phi(x)\right)-c(x) \phi(x)>0, \quad \text { for all } x \in A_{R} .
$$

Next we choose $a$ so that the function $\varphi(x)=a\left(e^{-\alpha r}-e^{-\alpha R}\right)$ satisfies $\varphi(x) \leq w_{1}$ in $|x|=R / 2$. We observe that

$$
\mathcal{M}_{\lambda, \Lambda}^{+}\left(D^{2} \varphi(x)\right)-c(x) \varphi(x)>0, \quad \text { for all } x \in A_{R}
$$

and $\varphi(x) \leq w(x)$ on $\partial A_{R}$. Using the comparison principle we find that $\varphi(x) \leq w(x)$ in $A_{R}$. Since $\varphi\left(x_{0}\right)=w\left(x_{0}\right)=0, \varphi$ can be viewed as a test function for equation (4.7) at $x_{0}$. Since $w$ is a supersolution we find that

$$
\mathcal{M}_{\lambda, \Lambda}^{+}\left(D^{2} \varphi\left(x_{0}\right)\right)-c\left(x_{0}\right) \varphi\left(x_{0}\right) \leq 0,
$$

which is impossible. This completes the proof. 


\section{ACKNOWLEDGMENTS}

The first author was partially supported by Fondecyt Grant \# 1030929 and FONDAP de Matemáticas Aplicadas, and the second author was partially supported by Fondecyt Grant \# 1040794 and Proyecto Interno USM \# 12.05.24.

\section{REFERENCES}

[1] A. Bensoussan, J.L. Lions, Applications of variational inequalities in stochastic control. Translated from the French. Studies in Mathematics and its Applications, 12. North-Holland Publishing Co., Amsterdam-New York, 1982. MR653144 (83e:49012)

[2] L. Bers. Isolated singularities of minimal surfaces. Ann. of Math. (2) 53, (1951), 364-386. MR0043335 (13:244c)

[3] I. Birindelli, F. Demengel, Comparison principle and Liouville type results for singular fully nonlinear operators. Ann. Fac. Sci. Toulouse Math. (6) 13 (2004), no. 2, 261-287. MR2126744 (2005k:35105)

[4] H. Brézis, L.Véron. Removable singularities for some nonlinear elliptic equations, Arch. Rational Mech. Anal. 75, (1980/81), 1-6. MR592099 (83i:35071)

[5] X. Cabré, L. Caffarelli. Fully Nonlinear Elliptic Equations, American Mathematical Society, Colloquium Publication, Vol. 43, 1995. MR1351007 (96h:35046)

[6] L. Caffarelli, B. Gidas, J. Spruck. Asymptotic symmetry and local behavior of semi-linear elliptic equations with critical Sobolev growth. Comm. Pure Appl. Math. 42, 3 (1989) 271-297. MR982351 (90c:35075)

[7] W. Chen, C. Li. Classification of solutions of some nonlinear elliptic equations. Duke Math. Journal, Vol. 3, No. 3, (1991), 615-622. MR1121147(93e:35009)

[8] C. Clemons, C. Jones, A geometric proof of the Kwong-McLeod uniqueness result, SIAM J. Math. Anal. 24 (1993) 436-443. MR:1205535 (94f:35047)

[9] A. Cutri, F. Leoni, On the Liouville property for fully nonlinear equations, Ann. Inst. H. Poincaré Analyse Non Linéaire 17 (2) (2000), 219-245. MR.1753094(2001h:35053)

[10] E. De Giorgi, G. Stampacchia. Sulle singolarità eliminabili delle ipersuperficie minimali. (Italian) Atti Accad. Naz. Lincei Rend. Cl. Sci. Fis. Mat. Natur. (8) 38 (1965) 352-357. MR0187158 (32:4612)

[11] P. Felmer, A. Quaas. On critical exponents for the Pucci's extremal operators. Ann Inst. Henri Poincaré, Analyse Non Linéaire 20, no. 5 (2003), pp. 843-865. MR1995504 (2004g:35083)

[12] P. Felmer, A. Quaas. Critical Exponents for Uniformly Elliptic Extremal Operators. Indiana Univ. Math. J. 55 (2006), no. 2, 593-629. MR2225447 (2007e:35084)

[13] B. Gidas, Symmetry properties and isolated singularities of positive solutions of nonlinear elliptic equations. Nonlinear partial differential equations in engineering and applied science (Proc. Conf., Univ. Rhode Island, Kingston, R.I., 1979), pp. 255-273, Lecture Notes in Pure and Appl. Math., 54, Dekker, New York, 1980. MR577096 (83m:35059)

[14] B. Gidas, J. Spruck, Global and local behavior of positive solutions of nonlinear elliptic equations. Comm. Pure Appl. Math. 34, (1981), 525-598. MR615628 (83f:35045)

[15] D. Gilbarg, N. S. Trudinger. Elliptic partial differential equation of second order, 2nd ed., Springer-Verlag, 1983. MR737190 (86c:35035)

[16] P.L. Lions, H. Ishi, Viscosity solutions of fully nonlinear second-order elliptic partial differential equations. Journal of Differential Equations 3, 26-78, (1990). MR.1031377(90m:35015)

[17] R. Jensen, The maximum principle for viscosity solutions of fully nonlinear second-order partial differential equations. Arch. Rat. Mech. Anal. 101, 1-27, (1988). MR 920674 (89a:35038)

[18] D. Labutin, Removable singularities for Fully Nonlinear Elliptic Equations, Arch. Rational Mech. Anal. 155 (2000) 201-214. MR1808368(2002a:35072)

[19] D. Labutin, Isolated singularities for Fully Nonlinear Elliptic Equations. Journal of Differential Equation 177 (2001), 49-76. MR.1867613 (2003a:35065)

[20] C. Pucci. Maximum and minimum first eigenvalues for a class of elliptic operators. Proc. Amer. Math. Soc. 17, (1966), 788-795. MR0199576 (33:7719)

[21] C. Pucci. Operatori ellittici estremanti, Ann. Mat. Pure Appl. 72 (1966), 141-170. MR0208150 $(34: 7960)$

[22] J. Serrin. Removable singularities of solutions of elliptic equations. Arch. Rational Mech. Anal. 17 (1964) 67-78. MR0170095 (30:336) 
[23] J. Serrin. Removable singularities of solutions of elliptic equations. II. Arch. Rational Mech. Anal. 20 (1965) 163-169. MR0186919 (32:4374)

[24] J. Serrin, H. Zou. Cauchy-Liouville and universal boundedness theorems for quasilinear elliptic equations and inequalities. Acta Math. 189 (2002), no. 1, 79-142. MR.1946918 (2003j:35107)

[25] L. Véron. Singularities of solutions of second order quasilinear equations. Pitman Research Notes in Mathematics Series, 353. Longman, Harlow, 1996. MR1424468 (98b:35053)

Departamento de Ingeniería Matemática, and Centro de Modelamiento Matemático, UMr2071 CNRS-UChile, Universidad de Chile, Casilla 170 Correo 3, Santiago, Chile

Departamento de Matemática, Universidad Santa María, Casilla: V-110, Avda. España 1680, Valparaíso, Chile 